\title{
Heat Transfer and Mode Transition for Laser Ablation Subjected to Supersonic Airflow *
}

\author{
Yi-Hui Huang(黄亿辉), Hong-Wei Song(宋宏伟) ${ }^{* *}$, Chen-Guang Huang(黄晨光) \\ Key Laboratory for Mechanics in Fluid-Solid Coupling Systems, Institute of Mechanics, \\ Chinese Academy of Sciences, Beijing 100190
}

(Received 29 May 2015)

\begin{abstract}
When laser ablation is subjected to supersonic flow, the influence mechanism of airflow on laser ablation behavior is still unclear. A coupled thermal-fluid-structure model is presented to investigate the influence of supersonic airflow on the development of a laser ablation pit. Results show that the aerodynamic convection cooling effect not only reduces the ablation velocity but also changes the symmetry morphology of the ablation pit due to the non-uniform convective heat transfer. Flow mode transition is also observed when the pit becomes deeper, and significant change in flow pattern and heat transfer behavior are found when the open mode is transformed into the closed mode.
\end{abstract}

PACS: 42.62.- b, 47.32.Ff, 44.20.+b

In the past decades, laser ablation of solids has drawn much attention owing to the wide application of laser in industrial and military fields..$^{[-8]}$ In some cases laser ablation is frequently subjected to airflow. For instance, in the process of laser cutting and drilling, crossjet airflow is utilized to blow away the ablation products and to avoid laser path shielding. ${ }^{[8]}$ Another example is in the military field, when a laser weapon is intercepting a high speed target which involves significant airflow. ${ }^{[9]}$ Although some research work has been carried out, the influence mechanism of airflow on laser ablation is still unclear due to the complex multi-physics coupling behavior of laser, airflow and structure. When a target is irradiated by a high-power laser, the temperature increases rapidly, and then melting, evaporation or sublimation occurs, the ablation pit appears on the surface. ${ }^{[11-14]}$ If the target is subjected to a tangential airflow, the ablation behavior may be different. Influence of airflow on the target manifests in several ways: aerodynamic force will blow away ablation products and will influence the deformation of the target, aerodynamic heat transfer will input or take away heat from the surface and influence the ablation behavior. Vice versa, the change in the ablation behavior will influence the airflow characteristics and will change the aerodynamic load distribution. Therefore, laser, airflow and target are coupled together and influence each other during the whole ablation process. The physical model is illustrated in Fig. 1, which describes the inflow and outflow of heat flux at the laser ablation boundary.

To reveal this coupling mechanism, some researchers ${ }^{[15,16]}$ presented coupled thermal-fluidstructure numerical models, while most of these mod- els did not take into account the ablation model. The main character of an ablation process is a moving boundary, at which heat transfer and phase transform take place, mathematically it is a Stephen problem. ${ }^{[17]}$ As the ablation pit evolves, the airflow pattern may significantly change and in reverse influence the development of the ablation pit. Therefore, the aerodynamic load is always changing and unknown in the process of laser ablation. In some research work the aerodynamic load is assumed to be the same, ${ }^{[18]}$ which is considered to be inaccurate. In this Letter, a coupled thermal-fluid-structure numerical model incorporating ablation is presented, which reflects interaction of airflow and target by using partition algorithm. The model can reflect how the ablation behavior and the airflow influence each other, and the numerical results reveal some new and interesting physical mechanisms.

In the model, some assumptions are made: (i) the laser is assumed to be of constant surface heat flux; (ii) the evaporation is the main mechanism of ablation, and evaporated gases are quickly removed by airflow; and (iii) the influence of evaporation on airflow is ignored, only considering the influence of ablation pit morphology on the airflow.

The heat conduction in the solid can be described by

$$
\rho c \frac{\partial T}{\partial t}=\frac{\partial}{\partial x_{i}}\left(\lambda \frac{\partial T}{\partial x_{i}}\right)
$$

According to Fig. 1, the thermal boundary condition is

$$
q_{\text {laser }}=\lambda \frac{\partial T}{\partial n}+\dot{m} Q_{\mathrm{abl}}+q_{\mathrm{conv}}+q_{\mathrm{rad}}
$$

\footnotetext{
*Supported by the National Natural Science Foundation of China under Grant Nos 11332011 and 11472276, the Project of the Chinese Academy of Sciences, and the Defense Industrial Technology Development Program.

** Corresponding author. Email: songhw@imech.ac.cn

(C) 2016 Chinese Physical Society and IOP Publishing Ltd
} 
where $T$ is the temperature, $\rho$ is the density, $c$ is the specific heat capacity, $\lambda$ is the thermal conductivity, $q_{\text {laser }}$ is the absorbed surface laser heat flux, which is assumed to be constant, $\dot{m}$ is the mass ablation rate on the surface, $Q_{\mathrm{abl}}$ is the latent heat of ablation, $q_{\mathrm{conv}}$ is the heat flux from the aerodynamic heat transfer of airflow, and $q_{\text {rad }}$ is the heat loss due to radiation.

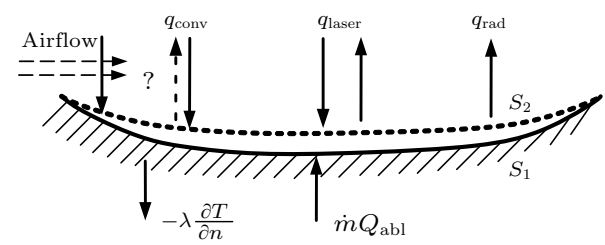

Fig. 1. Physical model of laser ablation subjected to the supersonic airflow.

The flow characteristics of the airflow can be described by the Navier-Stokes equations. For such a coupled problem, boundary conditions in fluid-solid interface are varying and unknown, while following the next rules

$$
\begin{aligned}
T_{\mathrm{s}} & =T_{\mathrm{f}}, \\
u_{\mathrm{s}} & =u_{\mathrm{f}}, \\
q_{\mathrm{conv}} & =q_{\mathrm{ae}},
\end{aligned}
$$

where $T_{\mathrm{s}}$ and $T_{\mathrm{f}}$ are the temperature of target and airflow in fluid-structure interface, respectively, $u_{\mathrm{s}}$ and $u_{\mathrm{f}}$ are the displacements at the boundary of solid field and fluid field, respectively, $q_{\text {conv }}$ is the aerodynamic heat flux to the solid due to airflow, and $q_{\text {ae }}$ is the heat flux of boundary layer in fluid-structure interface. Equations (3)-(5) guarantee temperature continuity, deformation compatibility and energy conservation at the fluid-structure interface. Therefore, the boundary conditions of solid field and fluid field are updated in each computational time step, reflecting the coupled behavior and mutual influence of airflow and structure during the ablation.

The ablation behavior and airflow characteristics are simulated by FEA (with an ANSYS code) and CFD (with a fluent code), respectively. By using a partition algorithm to control data transfer in the fluid-solid boundary, the coupled thermal-fluid-solid model is presented. To be consistent with Eqs. (3)(5), in each time-step, the CFD transfers wall heat flux to the FEA through the controlling interface, and the FEA transfers the temperature and ablation pit morphology to CFD. The staggered iteration and data exchange continues until convergence is reached, then moves to the next time-step. To ensure the convergence of computational results in each time-step, an implicit calculation method is employed. As meshes of solid and fluid in the fluid-structure interface do not match each other, the nearest neighbor interpolation is used during the data transfer.

Based on the model proposed above, the coupled thermal-fluid-structure ablation behavior of a plate target irradiated by high-power laser subjected to tangential supersonic airflow is investigated. The physical model basically follows Fig. 1. The Mach number of airflow is 4, and the spatial distribution of high power laser is in a Gaussian pattern, $q_{\text {laser }}=$ $q_{0} \exp \left(-2 x^{2} / r_{0}^{2}\right)$, where $q_{0}=6 \mathrm{MW} / \mathrm{m}^{2}$ and the laser spot radius $r_{0}=5 \mathrm{~mm}$. The thermal properties of the target material is listed in Table 1.

Table 1. Thermal properties of the target material.

\begin{tabular}{ccccc}
\hline $\begin{array}{c}\text { Density } \\
\rho\left(\mathrm{kg} / \mathrm{m}^{3}\right)\end{array}$ & $\begin{array}{c}\text { Evaporation temperature } \\
T_{\mathrm{s}}(\mathrm{K})\end{array}$ & $\begin{array}{c}\text { Latent heat of evaporation } \\
Q_{\mathrm{abl}}(\mathrm{kJ} / \mathrm{kg})\end{array}$ & $\begin{array}{c}\text { Specific heat } \\
c(\mathrm{~J} /(\mathrm{kg} \cdot \mathrm{K}))\end{array}$ & $\begin{array}{c}\text { Thermal conductivity } \\
\lambda(\mathrm{W} /(\mathrm{m} \cdot \mathrm{K}))\end{array}$ \\
\hline 8400 & 1623 & 309 & 550 & 18 \\
\hline
\end{tabular}

The numerical result shows that after irradiation for about $3.5 \mathrm{~s}$, the temperature at the spot center reaches the evaporation point $(1623 \mathrm{~K})$, the target material begins to be ablated, and the ablation pit appears. As irradiation duration accumulated, the ablation pit grows. An interesting phenomenon of flow pattern transition is observed at $4.0 \mathrm{~s}$ and $4.5 \mathrm{~s}$, as illustrated in Fig. 2. At $4.0 \mathrm{~s}$ the ablation pit is still shallow, the flow pattern is in a closed mode: airflow expands and separates in the front edge, one part flows to the bottom of the ablation pit then out of the pit. The remarkable characteristics of the closed mode flow are the higher velocity and no obvious vortex in the ablation pit. The aerodynamic pressure shows non-uniform distribution in the pit, at the front edge the pressure is much lower, whereas at the back edge aerodynamic pressure reaches the maximum due to the airflow compression. As the ablation pit becomes deeper, at $4.5 \mathrm{~s}$ the flow pattern changes into an open mode: airflow expands and separates in the front edge, then flows over the ablation pit. The main characteristics of the open mode are a lower velocity and the obvious vortex in the ablation pit compared with the closed mode. The aerodynamic pressure shows more uniformly distributed in the pit. According to the temperature profile, in the pit the fluid temperature is much higher than that in an open mode, due to the strong vortex and stagnant effect. The authors of Refs. [19,20] investigated the influence of cavity dimensions to the flow behaviors. Suppose that a high velocity fluid tangentially flows over a cavity with the dimension of length $L$ and height $H$, for $1 \leq L / H \leq 10$ the flow pattern is in an open mode, for $10<L / H<14$ it is a transition mode, whereas 
for $L / H \geq 14$ it typically demonstrates a closed mode flow. In the present study, at $4.0 \mathrm{~s} L / H$ is above 12 , whereas at $4.5 \mathrm{~s}$ it is about 5 . Therefore, the present numerical results are consistent with the previous theories.
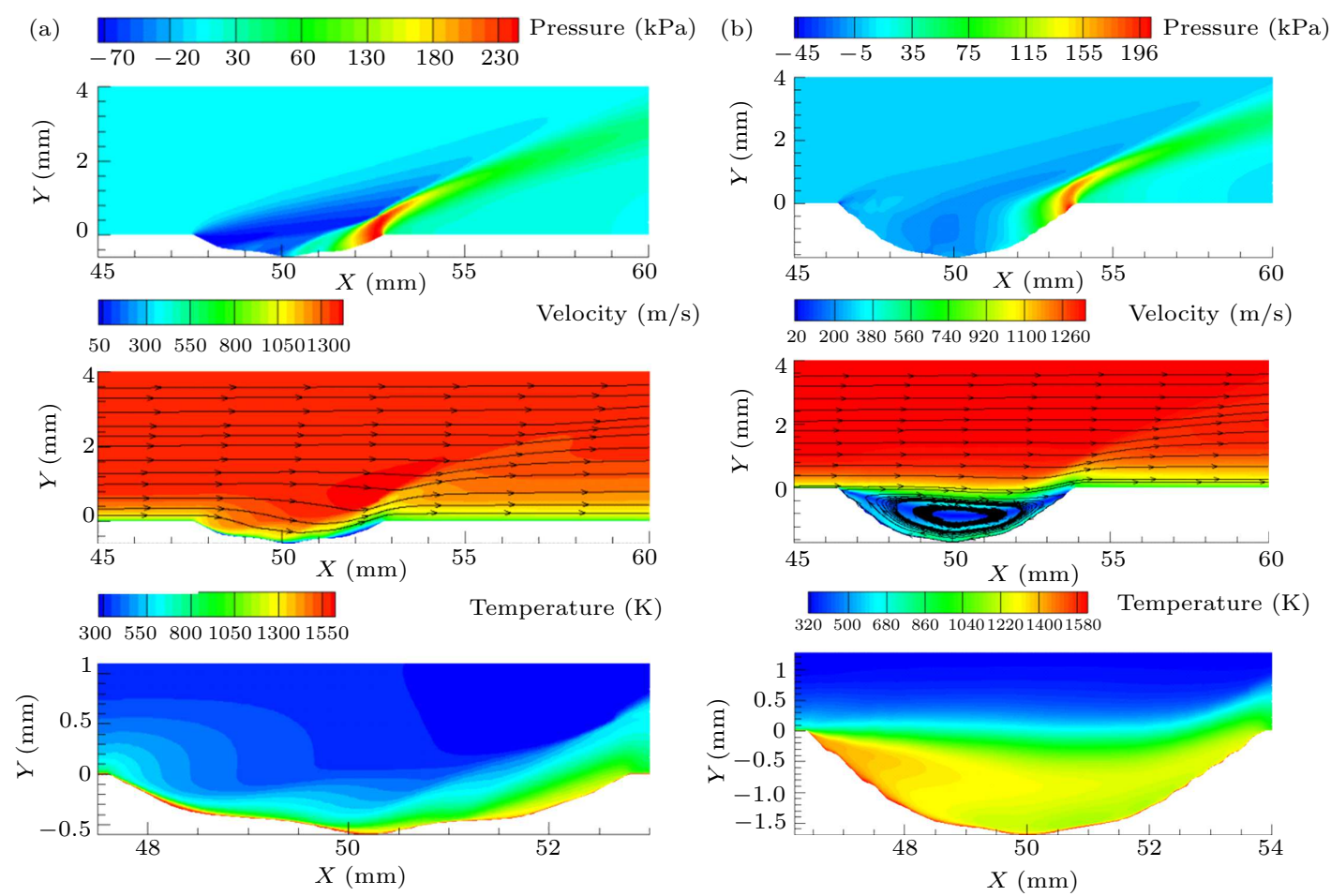

Fig. 2. Flow pattern transition from the closed mode to the open mode in adjacent time (a) $t=4 \mathrm{~s}$, closed mode (b) $t=4.5 \mathrm{~s}$, open mode.

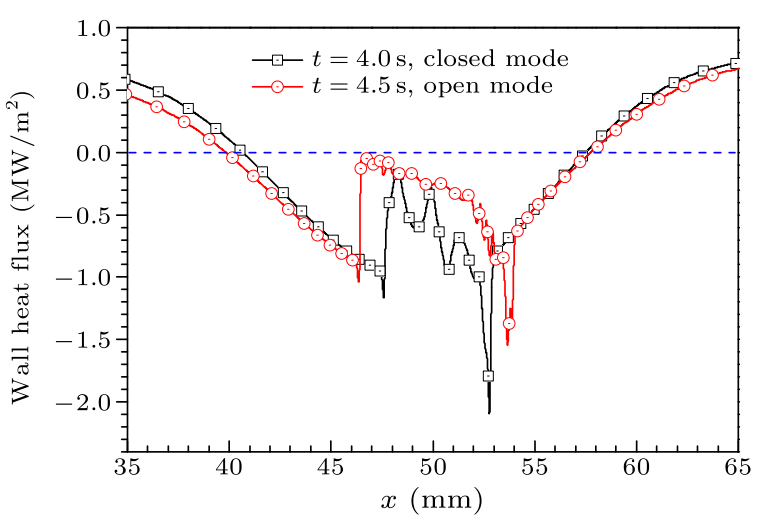

Fig. 3. Wall heat fluxes of the closed mode and the open mode.

The flow pattern also affects the convective heat transfer behavior along the pit boundary. As the adiabatic wall temperature of airflow is approximately $1200 \mathrm{~K}$, before the wall temperature of the target is heated to this level, the airflow demonstrates an aerodynamic heating effect, whereas when the wall temperature is above $1200 \mathrm{~K}$, it is an aerodynamic cooling effect. The wall heat flux shows an obvious difference as the flow mode changes from the closed mode to the open mode, which is shown in Fig. 3 . The negative value represents aerodynamic cooling, and the positive value represents aerodynamic heating. In the ab- lation pit, wall heat flux of the closed mode fluctuates obviously and shows strong cooling effect, whereas the cooling effect abated for an open mode, due to uniformity of airflow and lower velocity in the pit.

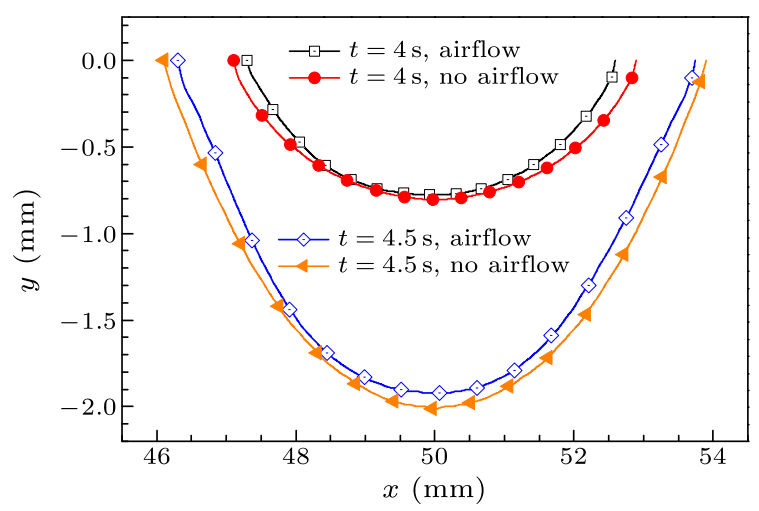

Fig. 4. Comparison of ablation pit morphologies for airflow and no airflow.

We have also simulated the case of laser ablation without airflow. It is shown that at first the temperature of the target subjected to airflow rises more quickly than that of without airflow due to aerodynamic heating, whereas when the temperature reaches $1200 \mathrm{~K}$ the tendency is in reverse since the aerodynamic cooling begins to play a role. Comparison of 
ablation pit morphology for the cases with airflow and without airflow is shown in Fig. 4. The main mechanism of heat transfer due to airflow in the ablation pit is aerodynamic cooling, therefore airflow will reduce the ablation velocity. For the case without airflow, the laser ablation pit shows perfect symmetry, whereas for the case with airflow it is asymmetry due to the nonuniform wall heat flux. The airflow cooling effect is lower in the front edge, therefore the ablation velocity here is higher than that of the back edge. Airflow reduces the ablation velocity as aerodynamic cooling plays a more important role, and it also changes the symmetry of ablation pit morphology due to the nonuniform wall heat flux.

In summary, we have presented a coupled thermalfluid-structure model to investigate the influence of supersonic airflow on laser ablation. The model is suitable for the case that the target is irradiated by a high-power laser and evaporation is the main ablation mechanism. Airflow convection heat transfer will reduce the ablation velocity due to the aerodynamic cooling effect. Airflow cooling not only reduces the ablation velocity but also changes the symmetry of ablation pit due to the non-uniformly distributed wall heat flux in the ablation pit. When the ablation pit becomes deeper, the flow mode changes from the closed mode to the open mode, the cooling effect decreases and becomes more uniform in the pit, and the influence of airflow begins to recede.

\section{References}

[1] Leitz K H, Koch H and Otto A 2012 Appl. Phys. A 106 885

[2] Cabanillas E D, Creus M F and Mercader R C $2005 \mathrm{~J}$. Mater. Sci. 40519

[3] Khersonsky A and Lee H 2000 Adv. Mater. Proce. 42

[4] Jia H N, Yang X J and Zhao W 2013 Chin. Phys. Lett. 30 044202

[5] Matsuda J and Utsuaii A 1998 Joining Mater. 712

[6] Zhao J H, Zhang G R and Liu X F 1996 Chin. J. High Press. Phys. 10262 (in Chinese)

[7] Wang W P, Tang X S and Gui Y Z 2001 Appl. Laser 21 319

[8] Yuan H, Zhao J H and Tan F L 2005 High Power Laser Part. Beams 15681 (in Chinese)

[9] Wang Z Y, Chen K and Zuo T 2000 Laser J. 2144

[10] Huang Y H, Song H W and Huang C G 2013 High Power Laser Part. Beams 252229 (in Chinese)

[11] Low D K Y, Li L and Byrd P J 2001 Opt. Lasers Eng. 35 149

[12] Ng G K L and Li L 2001 Opt. Laser Technol. 33393

[13] Low D K Y, Li L and Byrd P J 2002 J. Manuf. Sci. 124 852

[14] Dietrich J, Brajdic and Walther K 2008 Opt. Lasers Eng. 46705

[15] Zhang J and Huang C G 2007 High Power Laser Part. Beams 191818 (in Chinese)

[16] Zhang L, He J and Tan F L 2011 High Power Laser Part. Beams 23866 (in Chinese)

[17] Salva and Tarzia 2011 J. Math. Anal. 379240

[18] Shi W B, Li S X and Xiao Y 2010 High Power Laser Part. Beams 221215 (in Chinese)

[19] Selin A and Doyle K 2005 The 43rd AIAA Aerospace Sciences Meeting and Exhibition p 848

[20] Chung K M 1999 5th AIAA/CEAS Aeroacoustics Conference and Exhibition p 1909 